\title{
Stellar halos and the link to galaxy formation
}

\author{
Amina Helmi \\ Kapteyn Astronomical Institute \\ P.O. Box 800, 9700 AV Groningen, The Netherlands \\ email: ahelmi@astro.rug.nl
}

\begin{abstract}
I present a brief overview of how stellar halos may be used to constrain the process of galaxy formation. In particular, streams and substructure in stellar halos trace merger events but can also be used to determine the mass distribution of the host galaxy and hence put constraints on the nature of dark matter. Much of the focus of this contribution is on the Milky Way, but I also present an attempt to understand the kinematics of the globular cluster system of M31.
\end{abstract}

Keywords. Galaxy: halo; Galaxy: kinematics and dynamics; galaxies: formation; galaxies: halos; Local Group

\section{Introduction}

At first sight, it might appear perplexing to use stellar halos to understand the process of galaxy formation. For example, in a galaxy like the Milky Way more than $90 \%$ of the baryons (stars and gas) reside in the stellar disk and bulge/bar. The stellar halo contains likely less than $1 \%$ of the stellar mass (Helmi, 2008). How it can be claimed that such a component can be used to tackle galaxy formation?

While this argument is certainly valid and to address e.g. how most of the stars in the Milky Way formed one must study the Galactic disks and bulge, some of the physical processes associated to the build up of a galaxy as a whole leave also their imprints in the stellar halo. Let us therefore recall some specific properties of the halo that make it unique to probe certain aspects of galaxy formation.

First of all, stellar halos constitute a natural reservoir of merger debris. If mergers have been important in the build of the mass of the Galaxy (including dark matter), then the debris left behind is really the only way we have to access that history (Bullock \& Johnston, 2005; Deason et al., 2015).

Secondly, stellar halos are generally old, arguably contain the oldest stars we know of, not only in the field but also in globular clusters (Jofre \& Weiss, 2011; Leaman et al. 2013). This implies that by studying their properties we have access to the physical conditions present in the early Universe.

Finally, for example, by recovering the building blocks of the Milky Way we have access to both the properties of galaxies at the earliest epochs (long gone, perhaps even the first disks formed), as well as to the streams that are sensitive probes of the mass distribution and its assembly (Sanders \& Binney, 2013). These then help us pin down the nature of dark matter. Since dark matter is a fundamental ingredient of the current cosmological model, by studying its properties we also constrain galaxy formation in a broader sense.

\section{Substructure in stellar halos: history}

To study debris from merger events is very challenging, both observationally as well as theoretically. From the observational point of view one is limited in the case of external 

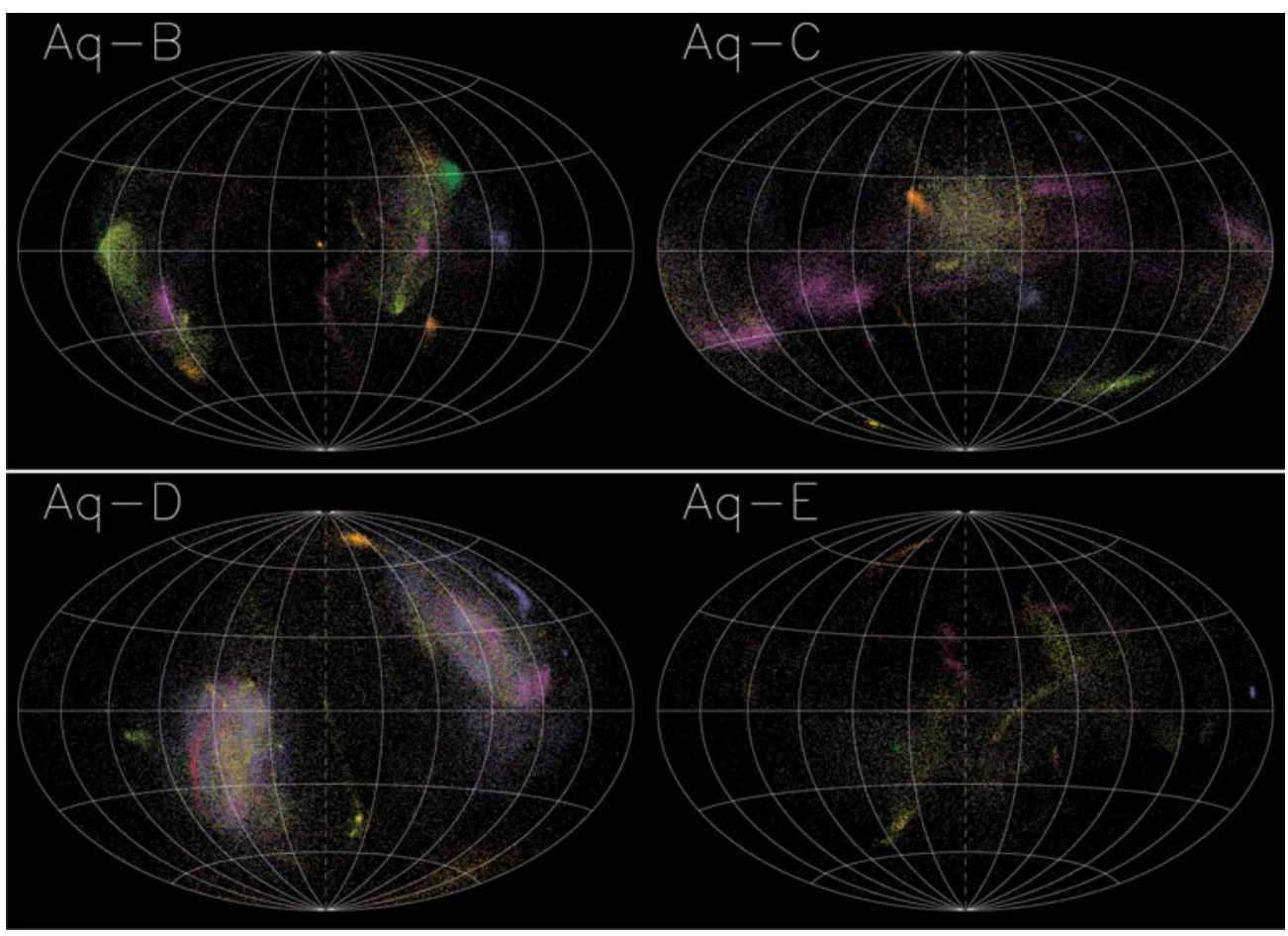

Figure 1. Sky distribution of stars in the Aquarius stellar halos, located at distances of 30-50 kpc from the "Sun". The different colours correspond to stars originating in different progenitors. Even more distant substructures are distributed anisotropically, and this should become apparent in the next generation of photometric surveys (see Helmi et al. 2011).

galaxies by the low surface brightness of the tidal features, and hence the requirements on the photometric quality are very demanding. Nonetheless, significant progress is being made and astounding panoramic views of stellar halos and debris are becoming steadily available (Martinez-Delgado et al. 2010; Janowiecki et al. 2010; Duc et al. 2015). From the numerical perspective, and because the stellar halo constitutes just a few percent of the stellar budget of a galaxy, simulations with very high resolution are required. This is why most of the studies of substructure in stellar halos in a cosmological framework have been done by "tagging" dark matter particles in a very high resolution dark matter only simulation (White \& Springel, 2000; De Lucia \& Helmi 2008; Cooper et al. 2010). The work of Cooper et al. stands out because of the exceedingly high resolution provided by the Aquarius simulations (Springel et al. 2008). Even selecting a merely $1 \%$ of the dark matter particles has allowed the authors to study not only the global properties of halos, but also the amount of substructure both in space and in velocity (Cooper et al. 2011; Helmi et al. 2011; Gomez et al. 2013).

One of the results that has come out of these cosmological simulations is that at large distances, and as shown in Fig. 1, stellar halos are very lumpy, and that stars are anisotropically distributed on the sky. Their distribution in fact reflects the infall pattern of the objects in which they originate, which relates to the filamentary nature of the large scale structure in which galaxies are embedded. This anisotropic distribution should become apparent in the next generation of full sky surveys of the Milky Way.

We can be more quantitative and measure the amount of spatial substructure present in these stellar halos, for example in the form of an RMS contrast $<\rho^{2}>^{1 / 2} /<\rho>$, and 
compare it to that of the Milky Way. Typically these halos depict a higher RMS contrast compared to a similar estimate obtained by using a sample of main sequence turn off stars in the SDSS over similar distances and area on the sky as for the simulations (Helmi et al. 2011). There are two points that need to be considered before firm conclusions about this difference can be drawn. Firstly, samples of main sequence turn off stars from surveys such as SDSS can suffer some amount of contamination from stars from e.g. the thick disk (and QSO's but this is easier to take into account because the distribution on the sky is much better known and relatively uniform). This contamination could provide as it were a screen on top of the underlying halo (whose relative normalisation and distribution on the sky are somewhat uncertain and model dependent), and thereby an RMS contrast $<\rho^{2}>^{1 / 2} /<\rho>$ that will necessarily be reduced since $\langle\rho\rangle$ becomes larger.

On the other hand, there is also a physical explanation, and that is that the modelled halos do not contain a smooth in-situ component. By construction, all halo stars are accreted in the Aquarius simulations. As argued by Helmi et al. (2011), a smooth component that accounts for $10 \%$ of the total stellar halo mass (or $30 \%$ in the inner $\sim 10$ $\mathrm{kpc}$ ), will provide enough of a screen to actually reduce the RMS contrast and bring it in agreement with the observations.

The high RMS contrast is evidently due to the very same asymmetry discussed earlier: It is the imprint of an anisotropic pattern that provides regions on the sky that are basically devoid of halo stars. This statistic is more sensitive than for example a measurement of the RMS obtained by comparison to a smooth halo model fitted to the data: $<\left(\rho-\rho_{m}\right)^{2}>^{1 / 2} /<\rho_{m}>$. Such a statistic will naturally lead to a smaller RMS value. This might partly explain why Bell et al. 2008 using also main sequence turn off stars in the SDSS found more consistency with the simulations of Bullock \& Johnston (2005).

Which of the above listed solutions will bring better agreement between cosmological models and the observations remains to be determined. Fortunately this will soon be possible with the ongoing sky surveys (PANSTARRS, DES, ATLAS) and also with the Gaia mission. If the halo has an important spatially smooth in-situ component then there should be little to no substructure in the kinematics in the form of moving groups or streams in the inner halo of the Galaxy. As Gomez et al. (2013) have shown, the Aquarius halos depict significant amounts of kinematic substructure even though they were built fully hierarchically and in a somewhat "chaotic" fashion. That substructure remains is not surprising as it is a consequence of volume conservation in phase-space, but it actually also implies that streams stars are not on exponentially diverging trajectories that would preclude detection of moving groups above the Poisson noise level.

\section{Streams as probes of mass distribution}

Streams stars are excellent probes of the gravitational potential in which they move because they map close to a single orbit (Sanders \& Binney, 2013). This characteristic, especially of thin streams, allows us in principle to determine the force field and its properties. Most of the work so far has focused on modelling the streams from the Sagittarius dwarf galaxy (e.g. Law \& Majewski, 2010), although more recently also other streams have been employed (e.g. Koposov et al. 2010; Kupper et al. 2015). At the moment the field is hungry for data, but this will soon become available thanks to the Gaia mission. In parallel it is important not to neglect the modelling aspect, as much of the work so far has been based on using a single stream, and typically with simplifying assumptions about the gravitational potential.

In an attempt to address these issues, Sanderson et al. (2015) have put forward a new method using multiple streams (integrated in a simple gravitational potential, as well 
as streams from the Aquarius simulations) to establish their ability to recover the true parameters of the underlying potential. Their method relies on the assumption that in the best potential, the streams will be most strongly clumped in the space of actions. Their work shows that even in the case of the hierarchically grown dark matter halos from the Aquarius simulations they are able to recover the characteristic parameters of the spherically averaged mass distribution very well, if full phase space coordinates are available for their stars (Sanderson, Hartke \& Helmi, in prep).

In the near future it will be important to incorporate more information (such as the angles, as argued by e.g. Sanders, 2014), but also establish if the shape or time-evolution are amenable to constraining, when realistic Gaia data samples (including errors) are available. Furthermore, exploration of a potential that has multiple components will also be necessary. The action-based approach seems very powerful, but will be expensive and difficult if the actions need to be computed for approximate potentials that may not provide uniformly good fits to the true potential at all locations in phase-space, or equivalently work equally well for all streams (although see Binney, 2012; Sanders \& Binney, 2015).

\section{Substructure and globular clusters}

The halo of our neighbour M31 is a great example of accretion in action. It provides us with a panoramic view of ongoing stellar halo assembly. Thanks to panoramic surveys such as PAndAS (McConnachie et al., 2009), many new globular clusters and dwarf galaxies in this system have been discovered (e.g. Mackey et al. 2010; Richardson et al. 2011). These have revealed their own surprises, such as what appears to be a giant disk structure of satellites, all rotating in the same sense (Ibata et al. 2013).

The spatial distribution and kinematics of the outer halo globular clusters of M31 have turned out to be extremely interesting. Often these halo clusters appear to be associated with streams and overdensities, possibly also dynamically because of their cold kinematics (Veljanoski et al. 2013). It is therefore very likely that these clusters have been accreted along with the progenitors of the streams seen in the halo of M31.

Also interesting is that as a population, the outer globular clusters depict rotation and that the axis of rotation is fairly aligned with the minor axis of M31's optical disk (Veljanoski et al. 2014). This is the true also for the inner globular clusters, although the rotation amplitude is smaller. Interestingly, the sense of rotation is the same as that of the giant disk of satellites, although the rotation axes are somewhat misaligned.

This large degree of coherence is astounding at first sight. It has been argued that it may be due to major gas-rich mergers in which many of the dwarfs could have formed (Hammer et al., 2013). However, we have noted earlier in this contribution that the distribution of stars in stellar halos in cosmological simulations is anisotropic and reflects the filamentary pattern of the cosmic web, along which satellites and building blocks have fallen in. In this sense it might perhaps not be so surprising to find coherence in the globular cluster population.

To explore to some extent the likelihood of finding rotation in a cosmological setting, Veljanoski \& Helmi (2016) have taken a simple approach. Using the Aquarius stellar halos, they have selected a subset of tagged particles and relabelled these as "globular clusters", an example of which is shown in Fig. 2. Using such Mock globular clusters systems, they asked: "how often is there a rotation signal in projection as large as observed in M31 globular cluster system, and how does this depend on the viewing angle"?

Fig. 3 shows some examples of how the rotation signal depends on viewing angle for the Mock systems in the Aquarius $\mathrm{C}$ halo. There are many angles for which the signal 


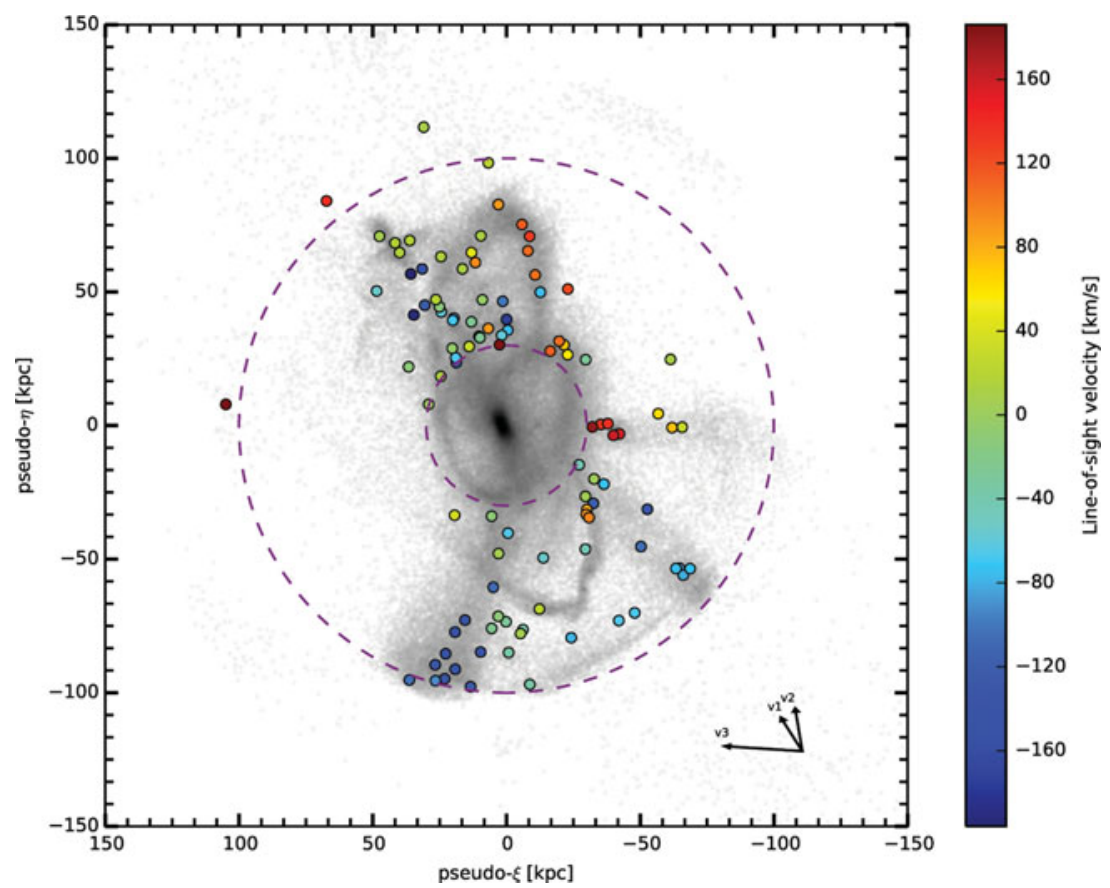

Figure 2. Sky distribution of stars (in grey) and "globular clusters" in the Aquarius-C halo, seen in projection from a distance of $1 \mathrm{Mpc}$. The colours indicate the line-of-sight velocities of the clusters, and for this particular viewing perspective the amplitude of the rotation signal is similar to that found in M31 (Veljanoski \& Helmi, in prep.).
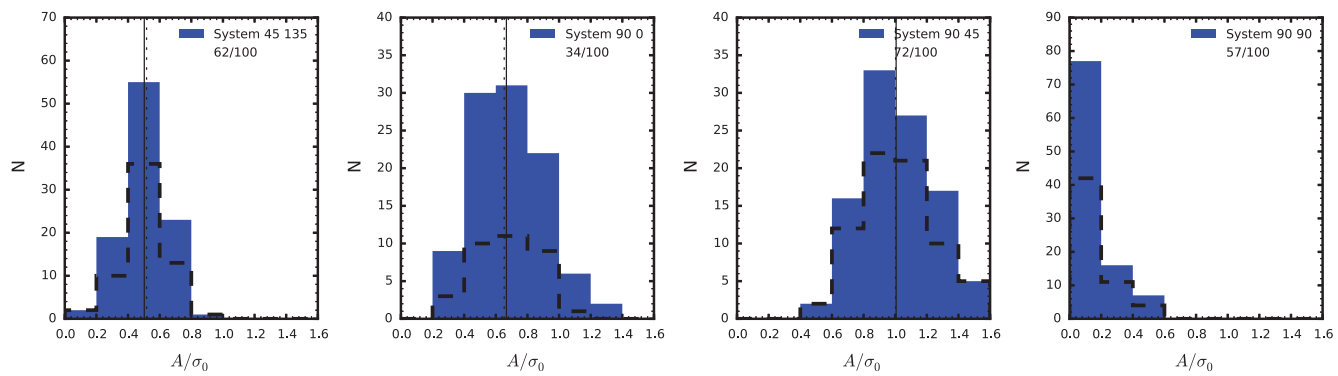

Figure 3. Distribution of the amplitude of the rotation signal $A / \sigma$ for Mock samples of globular clusters from the Aquarius $\mathrm{C}$ halo, for different viewing perspectives. The value found for M31 is $A / \sigma=0.67$ (Veljanoski \& Helmi, 2016).

has a lower amplitude than in M31 (left and right most panels), there are some for which the amplitude is larger (third panel). Averaged over all perspectives, the probability of having a kinematic ratio as big as in M31 or larger is 0.2 for the Aquarius C system.

The angle for which a high amplitude signal is observed corresponds to one in which the true rotation axis of the system is aligned within 30 degrees with the axis determined from the projected positions and velocities of the Mock globular clusters. The true rotation axis also aligns well with the axis of rotation of the whole stellar halo, again within 40 degrees. The question now is how is this correlated with the orientation or rotation axis of the disk. In the Aquarius simulations there is no disk component. However, what we do know about these simulations is that there is preferential infall along filaments, which implies that both the satellites as well as their debris, but also the gas that comes 
into the system are all reflecting similar directions of infall and rotation. Therefore, one expects that this gas, when it settles, ought to have the same rotation sense as that of the stellar halo (albeit a much higher amplitude because it has collapsed to the center), and therefore also the same as that of the globular clusters.

Essentially this seems to imply that we see high rotation in the globular cluster system of M31 because we are looking at M31's disk not too far from edge-on.

\section{Outlook}

In the coming decade we will be able to learn much more about the assembly history of stellar halos, both from the observational perspective as well as from the theoretical point of view. Observationally, we expect a revolution to take place with the Gaia satellite data, now that phase-space information for millions of halo stars will become available. This will allow us in the case of the Milky Way, to establish the relative importance of in-situ formation vs accretion, but also to recover whenever possible, the building blocks of the halo as well as determine their star formation and chemical evolution history using upcoming instruments such as WEAVE and 4MOST.

Going beyond our Galaxy will also be very important. Although for the Milky Way we will be able to study the progenitor systems in great detail, by studying other halos we get a broader overview of the process, which is likely to have been rather stochastic and time-dependent. To establish the ubiquity of accretion events, as well as to make quantitative comparisons to cosmological predictions, it is necessary to map the halos of hundreds to thousands of galaxies. This is challenging but can be extremely rewarding, and may be soon within reach.

From the theoretical perspective, higher resolution hydrodynamical simulations of systems like the Milky Way are urgently needed. The comparisons presented here, and most of those in the literature, rely on the ability of using dark matter only simulations to tag particles. But baryonic effects, such as the presence of a disk, might change both the shape of the stellar halos as well as the amount of debris present and the timescales of survivability (see e.g. Bailin et al. 2014). Cosmological simulations in which the stellar halo has at least $5 \times 10^{5}$ particles seem necessary, but will hopefully become feasible soon. Since different galaxies should have undergone different merger histories, quantitative comparisons with suites of simulations should allow us to probe some of the expected stochasticity of the process.

\section{Acknowledgements}

I am very grateful to my collaborators, especially the Virgo Consortium for the Aquarius simulations, Robyn Sanderson and Jovan Veljanoski. This work has been financially supported by ERC Starting Grant GALACTICA-240271, NOVA and an NWO-Vici grant.

\section{References}

Bailin, J., Bell, E. F., Valluri, M., et al. 2014, ApJ, 783, 95

Bell, E. F., Zucker, D. B., Belokurov, V., et al. 2008, ApJ, 680, 295

Bullock, J. S., \& Johnston, K. V. 2005, ApJ, 635, 931

Binney, J. 2012, MNRAS, 426, 1324

Cooper, A. P., Cole, S., Frenk, C. S., et al. 2010, MNRAS, 406, 744

Cooper, A. P., Cole, S., Frenk, C. S., \& Helmi, A. 2011, MNRAS, 417, 2206

Deason, A. J., Belokurov, V., \& Weisz, D. R. 2015, MNRAS, 448, L77 
De Lucia, G., \& Helmi, A. 2008, MNRAS, 391, 14

Duc, P.-A., Cuillandre, J.-C., Karabal, E., et al. 2015, MNRAS, 446, 120

Gómez, F. A., Helmi, A., Cooper, A. P., et al. 2013, MNRAS, 436, 3602

Hammer, F., Yang, Y., Fouquet, S., et al. 2013, MNRAS, 431, 3543

Helmi, A. 2008, A\&GARev, 15, 145

Helmi, A., Cooper, A. P., White, S. D. M., et al. 2011, ApJL, 733, L7

Jofré, P., \& Weiss, A. 2011, A\&3A, 533, A59

Ibata, R. A., Lewis, G. F., Conn, A. R., et al. 2013, Nature, 493, 62

Küpper, A. H. W., Balbinot, E., Bonaca, A., et al. 2015, ApJ, 803, 80

Koposov, S. E., Rix, H.-W., \& Hogg, D. W. 2010, ApJ, 712, 260

Law, D. R., \& Majewski, S. R. 2010, ApJ, 714, 229

Leaman, R., VandenBerg, D. A., \& Mendel, J. T. 2013, MNRAS, 436, 122

Mackey, A. D., Huxor, A. P., Ferguson, A. M. N., et al. 2010, ApJL, 717, L11

Martínez-Delgado, D., Gabany, R. J., Crawford, K., et al. 2010, AJ, 140, 962

McConnachie, A. W., Irwin, M. J., Ibata, R. A., et al. 2009, Nature, 461, 66

Janowiecki, S., Mihos, J. C., Harding, P., et al. 2010, ApJ, 715, 972

Richardson, J. C., Irwin, M. J., McConnachie, A. W., et al. 2011, ApJ, 732, 76

Sanders, J. L., \& Binney, J. 2013, MNRAS, 433, 1826

Sanders, J. L. 2014, MNRAS, 443, 423

Sanders, J. L., \& Binney, J. 2015, MNRAS, 447, 2479

Sanderson, R. E., Helmi, A., \& Hogg, D. W. 2015, ApJ, 801, 98

Springel, V., Wang, J., Vogelsberger, M., et al. 2008, MNRAS, 391, 1685

Veljanoski, J., Ferguson, A. M. N., Mackey, A. D., et al. 2013, ApJL, 768, L33

Veljanoski, J., Mackey, A. D., Ferguson, A. M. N., et al. 2014, MNRAS, 442, 2929

Veljanoski, J., \& Helmi, A., 2016, arXiv:1602.04018 (submitted to A\&A)

White, S. D. M., \& Springel, V. 2000, The First Stars, 327 